CORRECTION

https://doi.org/10.1038/s41586-018-0573-5

\title{
Author Correction: Global land change from 1982 to 2016
}

Xiao-Peng Song, Matthew C. Hansen, Stephen V. Stehman, Peter V. Potapov, Alexandra Tyukavina, Eric F. Vermote \& John R. Townshend

Correction to: Nature https://doi.org/10.1038/s41586-018-0411-9, published online 8 August 2018.

In Supplementary Table 1 of this Letter, owing to a copying error, the order of the numbers for nine countries with a name starting with the letter ' $C$ ' was incorrect. This meant that gross land-cover change estimates for these countries were inconsistent with net land-cover change estimates. The rest of the paper and its conclusions are not affected. For transparency, the Supplementary Information of this Amendment contains the old, incorrect Supplementary Table 1 (the errors occurred in rows 18-26, columns H, I, Q, R, Z and AA). Supplementary Table 1 has been corrected online.

Supplementary information is available in the online version of the Amendment. 